\title{
Fast colorimetric detection of albumin-to-creatinine ratio using paper-based analytical devices with alkaline picrate and Bromothymol Blue reagents
}

\author{
Nurrahmah Nurrahmah ${ }^{1}$, Kikie Trivia Amalia ${ }^{1}$, Hermin Sulistyarti ${ }^{1}$, Akhmad Sabarudin $^{1,2 *}$ \\ ${ }^{1}$ Department of Chemistry, Faculty of Science, Brawijaya University, Malang, Indonesia. \\ ${ }^{2}$ Research Center for Advanced System and Material Technology, Brawijaya University, Malang, Indonesia.
}

\begin{tabular}{l}
\hline ARTICLE INFO \\
\hline Received on: $27 / 05 / 2021$ \\
Accepted on: 09/09/2021 \\
Available Online: 05/01/2022 \\
\hline Key words: \\
albumin, creatinine, kidney, \\
nephropathy, paper-based \\
analytical devices.
\end{tabular}

\begin{abstract}
This work aimed to report the paper-based analytical devices (PADs) developed to determine the albumin-to-creatinine ratio (ACR) using the colorimetric method as a simple detection system. The detection of albumin (ALB) was based on the dye-binding reaction by immobilizing Bromothymol Blue (BTB) onto the PADs. The color change from yellow to blue was identified when ALB reacted with BTB. Creatinine (CRE) detection was carried out according to the Jaffe reaction by reacting $\mathrm{CRE}$ with alkaline picrate, which was immobilized onto the PADs, resulting in the color change from yellow to orange CRE-picrate complex. The intensity of the color formed is proportional to the concentration of ALB and CRE. The proposed method was successfully applied to detect ACR in synthetic urine samples with accuracy in the range of $77 \%-93 \%$ and relative standard deviations $<5 \%$. The detection limits for ALB and CRE were 2.4 and $5.3 \mathrm{mg} \mathrm{dl}^{-1}$, respectively. This device provides an inexpensive, simple, fast, disposable, and affordable tool for possible early detection of kidney damage because of nephropathy.
\end{abstract}

\section{INTRODUCTION}

Nephropathy is one of the global health problems, with a prevalence increasing every year. The main causes of nephropathy generally come from diabetes and hypertension, which can develop into kidney damage, chronic kidney disease (CKD), kidney failure, and even death (Mohart, 2013; Shiba and Shimokawa, 2011; Van Buren and Toto, 2011). The increasing number of hemodialysis patients showed increasing kidney failure because of nephropathy. According to the 2017 Global Burden of Disease, CKD ranks 12th and causes 1.1 million deaths worldwide. In the last 10 years, deaths from kidney disease have increased by $31.7 \%$, making kidney disease one of the leading causes of death, the fastest rising besides diabetes and dementia (Neuen et al., 2017).

\section{*Corresponding Author}

Akhmad Sabarudin, Department of Chemistry, Faculty of Science, Brawijaya University, Malang, Indonesia.E-mail: sabarjpn@ ub.ac.id
Early detection of nephropathy for diabetics and hypertension is urgently required to prevent kidney damage from getting worse. Nephropathy occurs when the albumin-tocreatinine ratio (ACR) in urine is $>30 \mathrm{mg} \mathrm{g}^{-1}$ or microalbuminuria is obtained [urine albumin (ALB) level is in the range of 30-299 $\mathrm{mg} / 24$ hour]. People with diabetes and hypertension, who suffer from nephropathy for at least 3 months, are referred to as CKD patients (Shaw and Cummings, 2012). If detected early and handled properly, the CKD patients with a urine ACR of 30-299 $\mathrm{mg} \mathrm{g}^{-1}$ and an eGFR of $60-89 \mathrm{ml} /$ minute/ $1.73 \mathrm{~m}^{2}$, the occurrence of kidney failure can be prevented (Han et al., 2015). Hence, fast laboratory testing and good disease management are needed.

Creatinine (CRE) and microalbuminuria detection are standard and widely used laboratory tests to determine kidney disorders in diabetic and hypertension patients. The amount of CRE excreted in urine indicates the condition of human kidneys. The collection of urine samples is effortless and not invasive, so it does not cause pain for patients. Urine contains proteins, end products of nucleic acid metabolism, metabolites (urea, uric acid, CRE, ammonia, and amino acids), 
various organic and inorganic salts, electrolytes, and many vitamins, hormones, enzymes, and other components as end products of the metabolism process. Through quantitative and qualitative monitoring of changes in urine composition, it is possible to obtain important diagnostic information of concerning kidney and urinary tract diseases and pathologies other organs such as the cardiovascular system, endocrine system, and metabolic system (Simerville et al., 2005). This urinalysis can also provide important information on disease progression, prognosis estimation, and treatment options (Jalal et al., 2017). However, concentrations of the urine component are influenced by diet, water intake, sweat, and other factors, so that the quantitative composition of urine varies significantly depending on the amount of urine excreted at a certain time. Consequently, measurement of the CRE concentration from a random urine sample as well as the concentration of the single urinary component CRE (Corder and Leslie, 2019; Fernandes et al., 2017; Krishnegowda et al., 2017; Ramanavicius, 2007; Yuen et al., 2004) does not provide accurate physiological information. Furthermore, CRE testing is considered less sensitive because when the kidney damage reaches $50 \%$, the CRE level just rises. For accurate analysis of urinary microALB, a 24-hours urine collection is needed for analysis, which requires considerable patient effort (Methven et al., 2010).

The effect of urine volume can be compensated by expressing the concentration of the desired urinary component as the number of analytes per total CRE. ALB index, which is the ratio of urinary ACR and is usually expressed as milligrams (mg) of ALB per gram ( $\mathrm{g}$ ) of CRE, is used as a substitute for this purpose. The urine ACR test avoids the volume influence problem on urine samples and does not require a 24-hours urine collection. Additionally, noninvasive sampling can be attributed to this method (Sabarudin, 2018). Accordingly, ACR is considered an accurate method for screening albuminuria. Besides being used to identify nephropathy, kidney function laboratory testing can also be applied to detect patients at high risk of hypertension and heart disease requiring more intensive care. However, the use of expensive autoanalyzer instrumentation in this method, which is usually only available in major/modern health facilities, can cause kidney disease more difficult to be detected early, particularly for patients away from the modern hospital.

Various analytical methods have been developed for the detection of ACR, including sequential injection analysis (Sabarudin, 2018; Siangproh et al., 2009), spectrophotometry (Schosinsky et al., 1987), turbidimetric immunoassay (Hong et al., 2016), and ELISA (Chapman et al., 2019). These methods employ modern instruments with high sensitivity and accuracy. However, the use of these instruments has limitations, such as high-cost analysis and time-consuming analysis, requires large amounts of reagents and samples and less portable and highpriced instrument, and requires a trained operator. Therefore, the development method, which is cheap, simple, fast analysis, disposable, and affordable, is needed. This need can be achieved using paper-based analytical devices (PADs).

Paper made from pure cellulose has chemical and physical properties, which are very suitable for application as
PADs (Yamada et al., 2015). The chromatographic paper does not need additives, and almost all impurities from the raw material are removed after the bleaching process. Cellulose is the primary component of the paper material, with an abundance of hydroxy $(-\mathrm{OH})$ groups and a small amount of carboxylic acid $(-\mathrm{COOH})$ groups on the surface (Alila et al., 2005). PADs were first introduced as an analytical tool with microfluidic patterns on paper to detect glucose and protein in artificial urine by Martinez et al. (2018). This device is very promising for use as a point of care diagnosis. PAD is a self-standing analysis system equipped with all the components needed to carry out an analytical assay, such as the sample transport system, sample pretreatment, reagents, and detection system.

The Jaffe method, even though it has been more than one century, is still used as a reference by most clinical laboratories for the quantitative determination of CRE. In this method, CRE reacts with alkaline picrate to form an orange-colored CRE-picrate complex whose color intensity is equivalent to the concentration of CRE in the sample (Delanghe and Speeckaert, 2011; Küme et al., 2017). ALB is generally determined using the dye-binding reaction method, resulting in color change which corresponds to ALB concentration when the dyes bind to this protein. Bromothymol blue (BTB) has higher sensitivity in comparison with Phenol red, Cresol red, and Thymol blue for detection of ALB using a visible spectrophotometric method (Suzuki, 2003).

The use of PAD to detect kidney disorders based on the multiple urinary components ACR has been reported. Disposable paper-based electrophoresis microchips with integrated pencildrawn electrodes (Chagas et al., 2016) provided a limit of detection (LOD) of $458 \mathrm{mg} \mathrm{dl}^{-1}$ for CRE and $262 \mathrm{mg} \mathrm{dl}^{-1}$ for ALB. Aerosolized deposition of polycaprolactone onto paperbased microfluidic tools (Heist et al., 2018) had an LOD of 50 $\mathrm{mg} \mathrm{dl}^{-1}$ for CRE and ALB. Paper-based colorimetry device for the determination of ACR using Bromocresol green and alkaline picrate reagents (Chaiyo et al., 2018) resulted in LOD of 5.4 and $7.1 \mathrm{mg} \mathrm{dl}^{-1}$ for CRE and ALB, respectively.

In this work, we developed colorimetric-based PADs for ACR detection. BTB immobilized in the PADs was used to determine ALB, resulting in a color change from yellow to blue. Moreover, CRE is determined using alkaline picrate, which results in a color change from yellow to orange. Although we used the same alkaline picrate reagent for CRE detection as reported by Chaiyo et al. (2018), the picric acid and $\mathrm{NaOH}$ concentrations used were different. Our optimized condition is better because it gives a sharper orange color (CRE-picrate complex) at the same creatine concentration so that it is easier to see with the naked eye. Using these reagents under optimum conditions, better sensitivity (LOD: 2.4 and $5.3 \mathrm{mg} \mathrm{dl}^{-1}$ for ALB and CRE, resp.) than other works reported so far (Chagas et al., 2016; Chaiyo et al., 2018; Heist et al., 2018) was achieved for ACR detection. The color changes were recorded using a digital camera, and their color intensities were measured using ImageJ software. The proposed method was then applied to the determination of ACR in the synthetic urine samples with satisfying results. 


\section{MATERIALS AND METHODS}

\section{Chemicals and apparatus}

The Xerox ColorQube 8580DN solid ink color printer (USA) was employed to prepare PADs (Fig. 1) using Whatman chromatographic paper No.1 (Whatman TM, GE Healthcare, UK). The digital drying oven (B-ONE, China) was used for posttreatment of the resulting PADs. The color changes on PADs were captured by the digital camera (Fujifilm X-A3, Japan) and further processed using ImageJ software to obtain the intensity of red, green, and blue (RGB) colors.

All chemicals used in this work were of the analytical reagent grade. Bovine serum albumin, $\mathrm{BTB}\left(\mathrm{C}_{27} \mathrm{H}_{28} \mathrm{Br}_{2} \mathrm{O}_{5} \mathrm{~S} / \mathrm{BTB}\right)$, $\mathrm{CRE}$, and sodium hydroxide for detection of $\mathrm{ACR}$ were purchased from Sigma-Aldrich (Singapore,) whereas picric acid $\left(\mathrm{C}_{6} \mathrm{H}_{3} \mathrm{~N}_{3} \mathrm{O}_{7}\right)$ was obtained from Smart-Lab, Indonesia. For preparation of artificial urine solution, citric acid, urea, lactic acid, potassium dihydrogen phosphate, dipotassium hydrogen phosphate, sodium chloride, sodium sulfate, sodium bicarbonate, ammonium chloride, calcium chloride, and magnesium sulfate were obtained from Sigma-Aldrich (Singapore). All chemicals were prepared in distilled water except BTB, which requires ethanol (Merck, Indonesia) for dissolution.

\section{Design and fabrication of $\mu$ PADs}

The PAD pattern was designed using the CorelDraw X7 software, which consists of two detection zones for ALB and CRE. Each detection zone had a diameter of $10 \mathrm{~mm}$, with PADs dimensions of $42 \times 23 \mathrm{~mm}$. The design (Fig. 1) was printed on Whatman chromatographic paper No.1 using the Xerox ColorQube 8580DN printer equipped with a CMYK (Cyan, Magenta, Yellow, Key) wax ink cartridge. The printed devices were placed in the oven at $120^{\circ} \mathrm{C}$ for 3 minutes to allow the wax to melt and penetrate PADs, generating the hydrophobic barrier on both sides of the paper. The backside of PADs was coated with transparent tape to prevent leakage of solution through the device and avoid paper bending when the reagents were dropped on the paper.

\section{Colorimetric detection of ALB and CRE}

The colorimetric detection procedure is shown schematically in Figure 2. The BTB and the mixture of picric acid with $\mathrm{NaOH}$ (alkaline picrate) reagents were dropped on ALB and CRE detection zones until they filled the circle zone area as indicated by the formation of a yellow color. Then, the standard solutions or samples were dropped on each detection zone and allowed to change color from yellow to blue for ALB and yellow to orange for CRE detection. These color changes were captured using the digital camera, and the resulting images were analyzed using Image J software to determine the RGB color intensity of ALB-BTB and CRE-alkaline picrate. The color intensity of the formed complexes can be represented by only one complementary color, which showed a linear trend as the concentration of the analytes increased. In this work, the red intensity was used to determine the ALB concentration, and green intensity was chosen to determine the CRE concentration. Then, the color intensity was corrected into $\Delta$ intensity (absolute value) by subtracting the blank intensity with red or green intensity, reflecting the actual intensity of the sample.

When photographing color changes using a digital camera (Fig. 2D), the PAD is adjusted to a fixed position and condition. The PAD is put inside a black controlled-light box with dimensions of $19 \mathrm{~cm}$ length $\times 14 \mathrm{~cm}$ width $\times 23 \mathrm{~cm}$ height; the top has a hole with a diameter of $5 \mathrm{~cm}$ as a place to insert the camera lens. At the bottom of the box, there is a place to put a PAD with a height of $3 \mathrm{~cm}$, and the distance between the PAD and the camera lens is $18 \mathrm{~cm}$. An LED light (4 watts, 6,500 K, 330 $\operatorname{lm}, 30 \mathrm{~mA}$ ) is placed inside the box whose position is fixed not to block the camera lens. Digital camera with fixed lens 16.0-50.0 $\mathrm{mm}$ (Fujifilm X-A3) is set as follows: focal length $16.0 \mathrm{~mm}$ with ISO 200.

\section{Synthetic urine samples}

The synthetic sample solution was made by mixing ALB and $\mathrm{CRE}$ at certain concentrations in artificial urine solution as

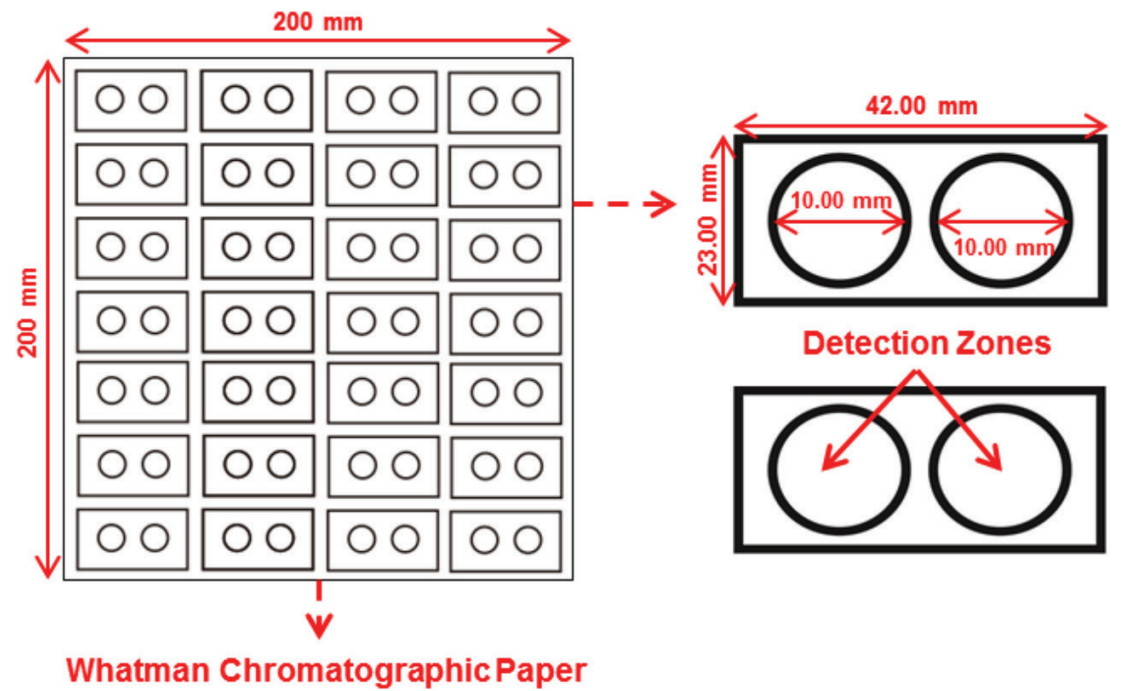

Figure 1. Design of the PADs. 


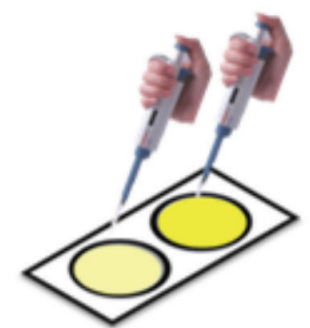

(A)

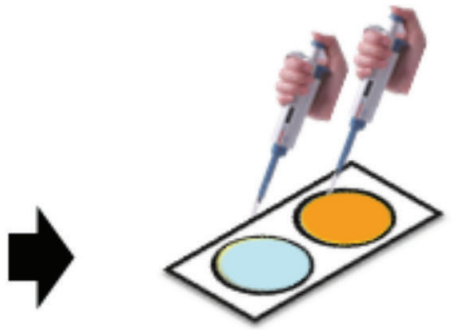

(B)

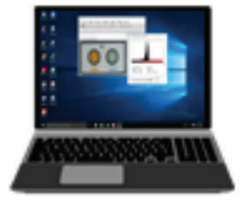

(E)
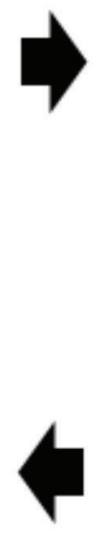

(D)

Figure 2. Colorimetric detection procedure for ALB and CRE detection. (A) Dropping BTB and alkaline picrate reagents to ALB and CRE detection zones. (B) Dropping standard solutions or samples to ALB and CRE detection zones. (C) The resulting color changes: blue for ALB and orange for CRE. (D) Capturing the color changes with the digital camera. (E) Analyzing RGB intensity using ImageJ software.

Table 1. Composition of artificial urine solution.

\begin{tabular}{cc}
\hline Components & Concentration $(\mathbf{m M})$ \\
\hline Citric acid & 2 \\
Urea & 170 \\
Uric acid & 0.4 \\
Lactic acid & 1.1 \\
Potassium dihydrogen phosphate & 7 \\
Dipotassium hydrogen phosphate & 7 \\
Sodium chloride & 90 \\
Sodium sulfate & 10 \\
Sodium bicarbonate & 25 \\
Ammonium chloride & 25 \\
Calcium chloride & 2.5 \\
Magnesium sulfate & 2 \\
\hline
\end{tabular}

shown in Table 1 (Brooks and Keevil, 1997; Martinez et al., 2007) at four different ALB index (ACR) concentrations as follows: synthetic sample 1 (CRE $100 \mathrm{mg} \mathrm{dl}^{-1}$; ALB $30 \mathrm{mg} \mathrm{dl}^{-1}$ ), synthetic sample 2 (CRE $100 \mathrm{mg} \mathrm{dl}^{-1}$; ALB $50 \mathrm{mg} \mathrm{dl}^{-1}$ ), synthetic sample 3 (CRE $100 \mathrm{mg} \mathrm{dl}^{-1}$; ALB $100 \mathrm{mg} \mathrm{dl}^{-1}$ ), and synthetic sample 4 (CRE $100 \mathrm{mg} \mathrm{dl}^{-1}$; ALB $400 \mathrm{mg} \mathrm{dl}^{-1}$ ). These compositions correspond to the ALB index of $300,500,1,000$, and 4,000 for synthetic urine samples $1,2,3$, and 4 , respectively.

\section{RESULTS AND DISCUSSION}

\section{Effect of reagent and sample volumes on the color intensity}

Investigation of the optimum reagent volume was carried out to determine the amount of reagent distributed in the PADs so that the color change obtained in the detection zone could be observed evenly and homogeneously. The reagent volume was varied from 1 to $3 \mu \mathrm{l}$, and the result is shown in Figure 3. The color intensity increased with increasing reagent volume and reached optimum when $2 \mu \mathrm{l}$ of each BTB and alkaline picrate was used for $\mathrm{ALB}$ and $\mathrm{CRE}$ detection, respectively. This selection was indicated by the blue (ALB-BTB) and orange (CRE-alkaline picrate) color formation, which provided the highest $\Delta$ color intensity and color distribution evenness in the detection zone. However, reagent volume excess $(>2 \mu \mathrm{l}$ ) would result in the reagent passing through the hydrophobic barrier of the detection zone.

The optimization of sample volume is required to find the desired amount of sample that can react with reagents, resulting in color change evenness in the detection zone without upward or downward bending of the PADs. In this work, the sample volume was studied from 2 to $6 \mu$, and the results are shown in Figure 4. The $\Delta$ color intensity increased as the sample volume rose. It was found that the blue color compound (ALB-BTB) and orange color complex (CRE-alkaline picrate) reached the highest $\Delta$ intensity accompanied by excellent color distribution in the detection zone when the sample volume was $4 \mu \mathrm{l}$. Accordingly, this volume was chosen for further experiments. The sample volume of $>4 \mu \mathrm{l}$ could 
(A)

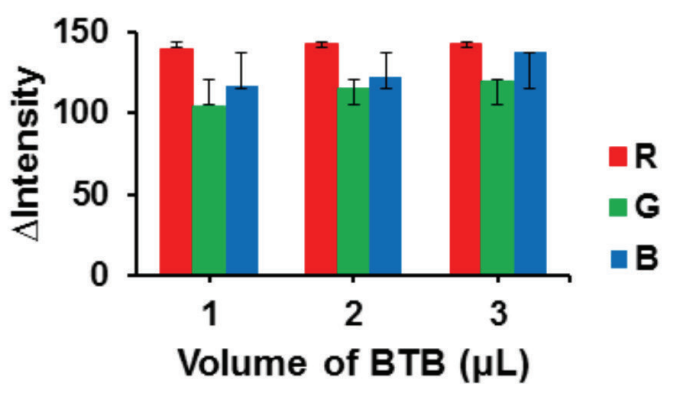

(B)

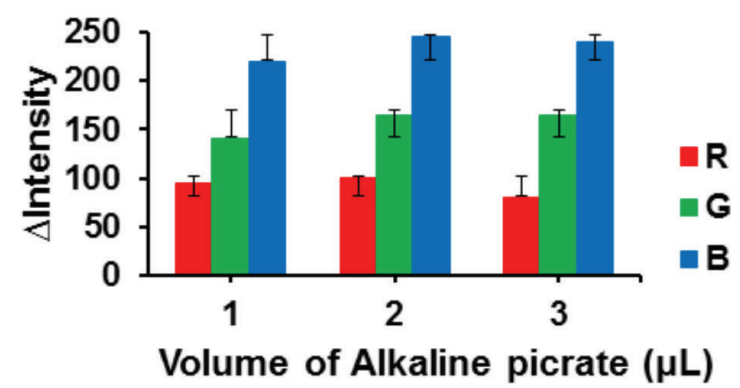

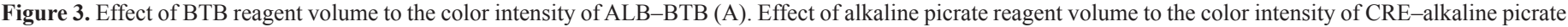
(B). Condition: volume of sample was $4 \mu \mathrm{l}$; ALB concentration was $100 \mathrm{mg} \mathrm{dl}^{-1}$; CRE concentration was $100 \mathrm{mg} \mathrm{dl}^{-1}$.

(A)

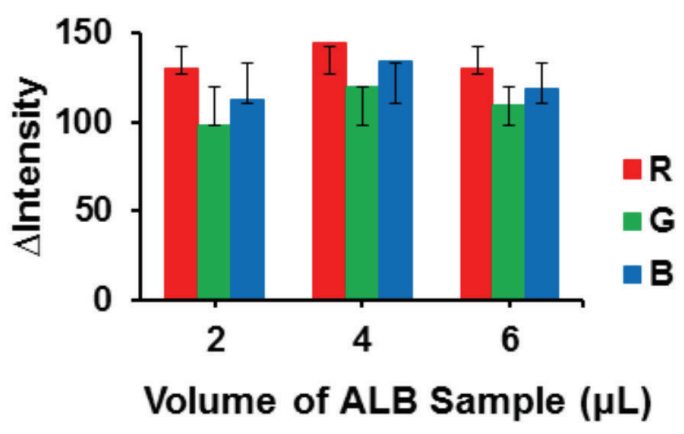

(B)

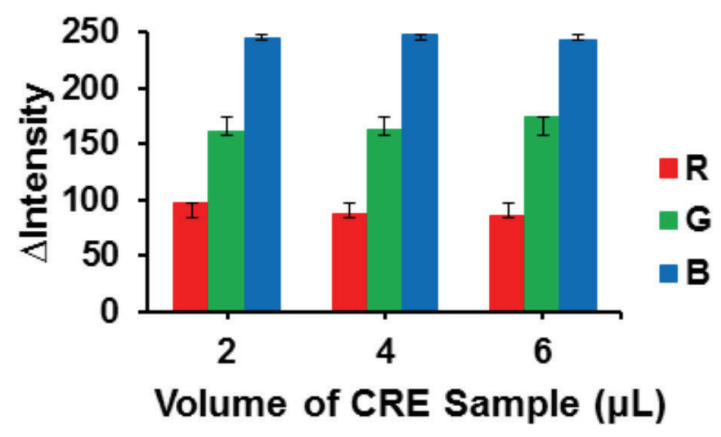

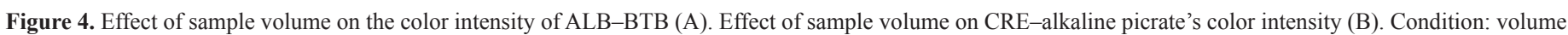
of reagent was $2 \mu \mathrm{l}$; ALB concentration was $100 \mathrm{mg} \mathrm{dl}^{-1}$; CRE concentration was $100 \mathrm{mg} \mathrm{dl}^{-1}$.

not be applied since the excess amount of solution passed through the hydrophobic barrier of circle detection zones.

\section{Effect of reagent concentration on the color intensity}

BTB concentration affects the color change for ALB detection, while alkaline picrate concentration affects the color change for CRE detection. Optimization of reagent concentration was carried out to obtain reagent composition that resulted in sharp color changes in the detection zone of PADs. The BTB concentration was varied from 0.0004 to $0.004 \mathrm{M}$, while the concentration of picric acid was studied from 0.01 to $0.05 \mathrm{M}$, and the concentration of $\mathrm{NaOH}$ for conditioning picric acid was investigated from 1 to $3 \mathrm{M}$. For picric acid, concentration $>0.05$ $\mathrm{M}$ could not be tested because it exceeded its solubility limit (saturated) (Cook et al., 1975).

As shown in Figure 5 (A), the results showed that the $\Delta$ intensity increased with increasing reagent concentrations. The optimum concentration of BTB was $0.0008 \mathrm{M}$ because it provided sharper and faster blue color change (ALB-BTB) in comparison with $0.004 \mathrm{M}$. In Figure 5 (B and $\mathrm{C}$ ), the $\Delta$ intensity of CREalkaline picrate increased with increasing picric acid and $\mathrm{NaOH}$ concentrations. Thus, $0.05 \mathrm{M}$ picric acid in $3 \mathrm{M} \mathrm{NaOH}$ (alkaline picrate) was selected for the further experiment because it resulted in sharper and faster orange color changes in the detection zone of PADs when more CRE was dropped.

\section{Effect of reaction time on the color intensity}

Investigation of the optimum reaction time was studied to find the appropriate reaction time so that the formation of faded colors in the detection zone of PADs could be avoided. PADs were photographed using a digital camera with reaction times varying from 5 to 75 minutes. In Figure 6, the results showed that the $\Delta$ color intensity of ALB-BTB (blue) and CRE-alkaline picrate (orange) in the PADs detection zone tended to be constant from 5 to 20 minutes. Conversely, at the reaction time $>20$ minutes, the $\Delta$ intensity decreased and the color in the PADs detection zone faded. Therefore, the optimum reaction time for detection of ALB and CRE can be carried out in the range of 5-20 minutes.

\section{The analytical figure of merits}

In this work, linearity is an important part of evaluating a method to determine the concentration of analytes with high accuracy using PADs. Additionally, the linearity is constructed according to the optimum conditions obtained. Due to the best linearity, the red intensity was applied to construct the calibration curve of ALB, whereas green intensity was used to prepare the calibration curve of CRE. Good linearity of ALB concentration was achieved in the range of $10-100 \mathrm{mg} \mathrm{dl}^{-1}$ (Fig. 7C) as indicated by the correlation coefficient $\left(R^{2}\right)$ of 0.9948 , whereas the linear range for the determination of CRE could be constructed from its concentration from 10 to $100 \mathrm{mg} \mathrm{dl}^{-1}$ (Fig. 7D), resulting in $R^{2}$ of 
(A)

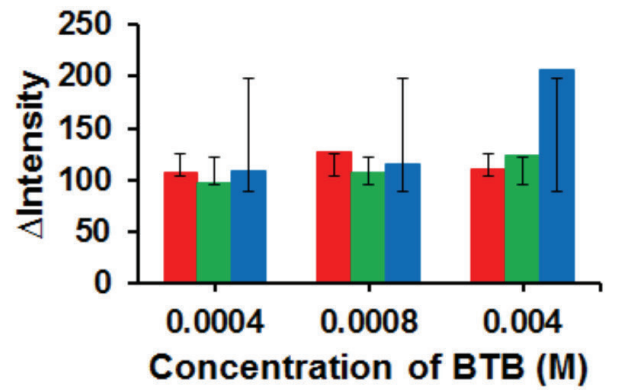

(B)

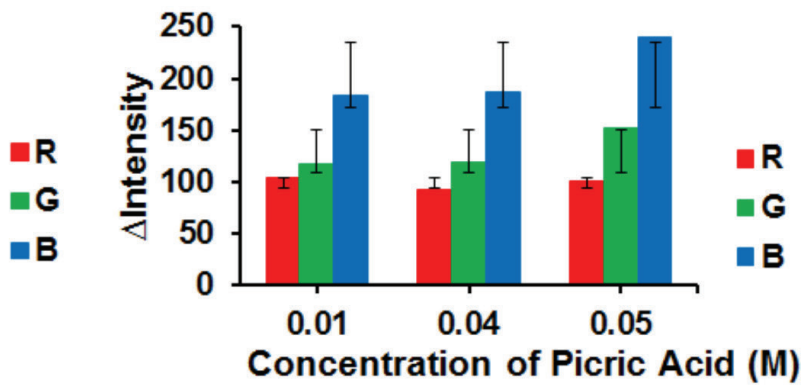

(C)

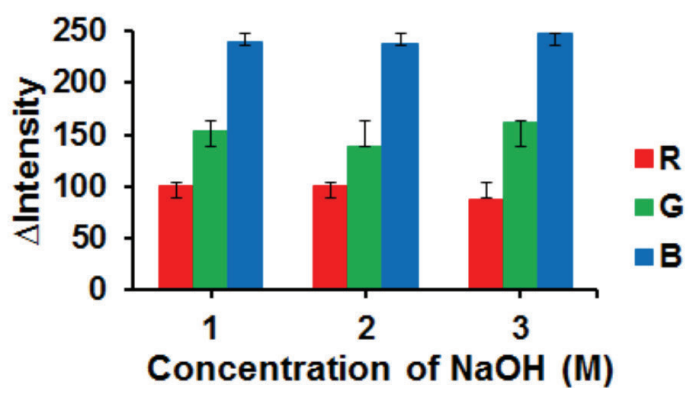

Figure 5. Effect of reagent concentration on the color intensity of BTB (A)., Concentration of picric acid (B)., Concentration of NaOH (C). Condition: volume of reagent was $2 \mu \mathrm{l}$; the volume of sample was $4 \mu \mathrm{l}$; ALB concentration was $100 \mathrm{mg} \mathrm{dl}^{-1}$; CRE concentration was $100 \mathrm{mg} \mathrm{dl}{ }^{-1}$. For (B), concentration of $\mathrm{NaOH}$ was fixed at $3 \mathrm{M}$. For (C), concentration of picric acid was fixed at $0.05 \mathrm{M}$.

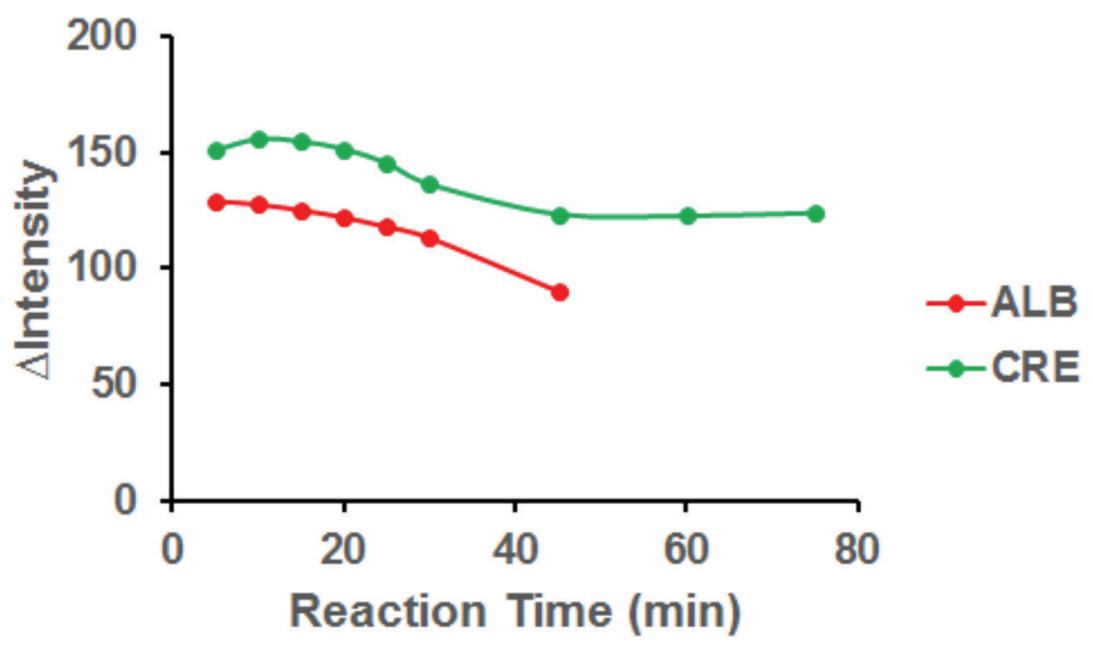

Figure 6. Effect of the reaction time to color intensity for ALB and CRE detection. Condition: volume of reagent was $2 \mu 1$; the volume of sample was $4 \mu \mathrm{l}$; concentration

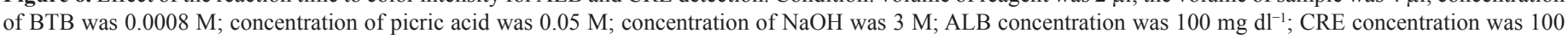
$\mathrm{mg} \mathrm{dl^{-1 }}$.

0.9723. The LOD of ALB and CRE were calculated according to $3 \mathrm{x}$ standard deviation $(3 \sigma)$ of the blank intensity of PADs for 10 measurements. After plotting to each linear equation, the LOD for ALB and CRE was found to be 2.4 and $5.3 \mathrm{mg} \mathrm{dl}^{-1}$, respectively.

The reproducibility was estimated under optimum conditions for five measurements of ALB $\left(40 \mathrm{mg} \mathrm{dl}^{-1}\right)$ and CRE (90 $\mathrm{mg} \mathrm{dl}^{-1}$ ). Excellent reproducibility could be achieved as relative standard deviations (RSD) of $0.27 \%$ and $0.24 \%$ were obtained for ALB and CRE, respectively.

\section{Determination of ACR in synthetic urine samples}

ACR curve was constructed under optimum condition using ALB concentration of $30-500 \mathrm{mg} \mathrm{dl}^{-1}$, while the CRE concentration was kept at $100 \mathrm{mg} \mathrm{dl}^{-1}$, corresponding to the ALB index (ACR) of 300 to $5,000 \mathrm{mg} \mathrm{g}^{-1}$ as calculated according to Equation (1) as follows:

$$
\operatorname{ACR}\left(\mathrm{mg} \mathrm{g}^{-1}\right)=\frac{[\mathrm{ALB}] \text { in } \mathrm{mg} \mathrm{dL}^{-1}}{[\mathrm{CRE}] \text { in } \mathrm{mg} \mathrm{dL}^{-1}} \times 100
$$


(A)
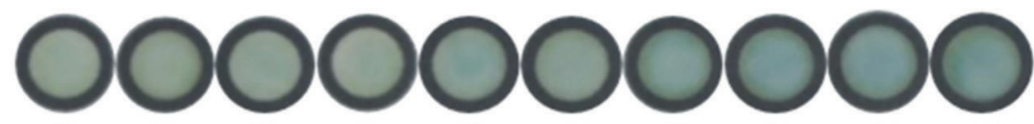

1020

$30 \quad 40$

50

60

$70 \quad 80 \quad 90$

$100 \mathrm{mg} \mathrm{dL}^{-1}$ ALB-BTB

(B)

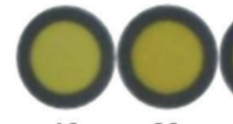

10

20
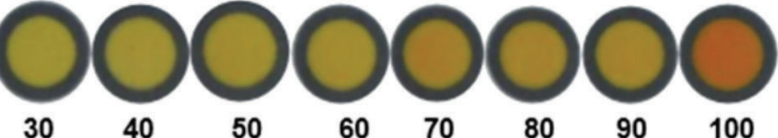

$100 \mathrm{mg} \mathrm{dL}^{-1}$ CRE-Alkaline picrate
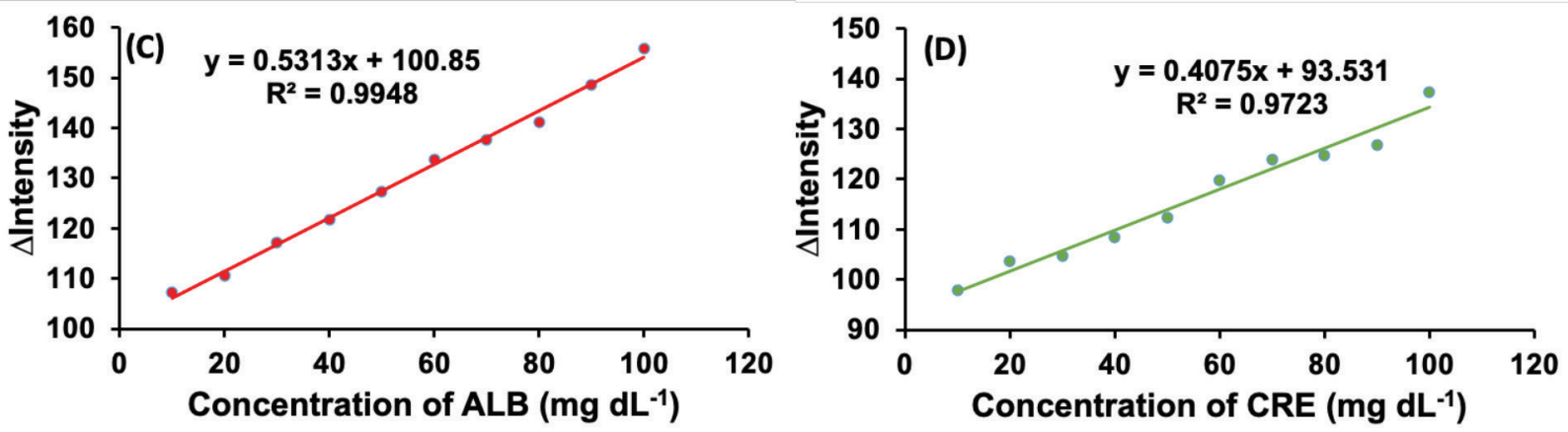

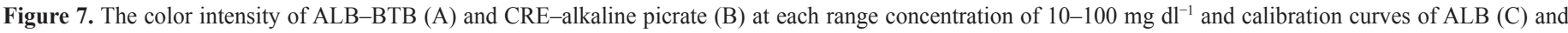

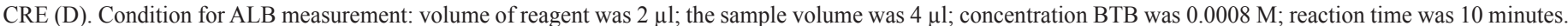
Condition for CRE measurement: concentration of picric acid was $0.05 \mathrm{M}$; concentration of $\mathrm{NaOH}$ was $3 \mathrm{M}$; other conditions were similar to ALB measurement.

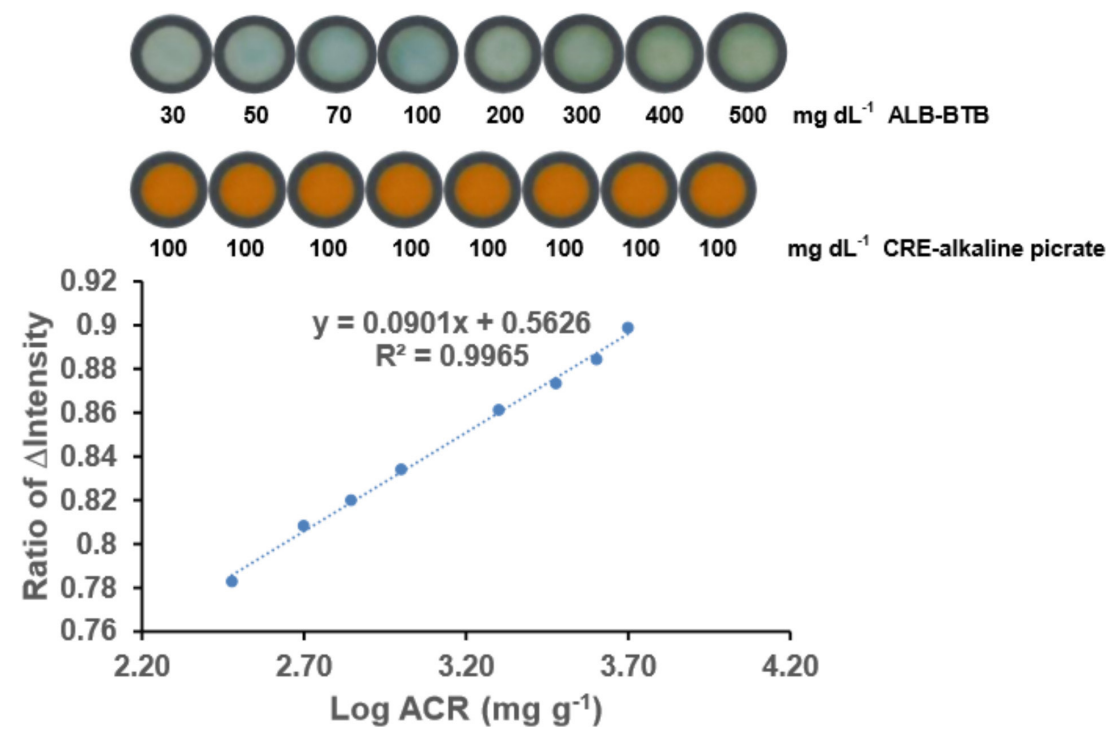

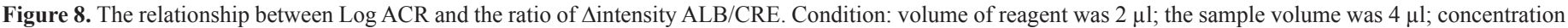
BTB was $0.0008 \mathrm{M}$; concentration of picric acid was $0.05 \mathrm{M}$; concentration of $\mathrm{NaOH}$ was $3 \mathrm{M}$; reaction time was 10 minutes; $n=3$.

Then, the logarithmic of ACR was plotted against the $\Delta$ intensity ratio $\mathrm{ALB} / \mathrm{CRE}$, resulting in excellent linearity with a correlation coefficient $\left(R^{2}\right)$ of 0.9965 , as shown in Figure 8 . The relatively wide linear range was sufficient to allow the proposed method to be used as an alternative method to determine the albuminuria levels. Additionally, this proposed device demonstrates a paper-based sensing capability to measure ACR easily.

The application of this method for ACR detection using synthetic samples was carried out to ascertain the performance of the proposed PADs. By calculating the
$\Delta$ intensity ratio of $\mathrm{ALB} / \mathrm{CRE}$ in the synthetic samples and plotting the results into the linear equation (see Fig. 8), the ACR values could be obtained. Thus, the albuminuria levels, such as ACR urine $<30 \mathrm{mg} \mathrm{g}^{-1}$ (normal albuminuria), $>30-300 \mathrm{mg} \mathrm{g}^{-1}$ (microalbuminuria), and $>300 \mathrm{mg} \mathrm{g}^{-1}$ (macroalbuminuria), can be estimated.

The analytical results of synthetic urine samples are shown in Table 2. The accuracy of ACR measurement was in the range of $77 \%-93 \%$, while the accuracy of ALB measurement was in the range of $90 \%-98 \%$. The RSD for both ACR and ALB measurements were 
Table 2. Analytical results of synthetic urine samples ${ }^{\mathrm{a}}$ using PADs $(n=3)$

\begin{tabular}{|c|c|c|c|c|c|c|}
\hline \multirow[b]{2}{*}{ Sample } & \multicolumn{2}{|c|}{ Added } & \multicolumn{2}{|c|}{ Found } & \multicolumn{2}{|c|}{ \%accuracy } \\
\hline & $\begin{array}{c}\text { ALB } \\
\left(\mathrm{mg} \mathrm{dl}^{-1}\right)\end{array}$ & $\begin{array}{c}\text { ACR } \\
\left(\mathrm{mg} \mathrm{g}^{-1}\right)^{\mathrm{b}}\end{array}$ & $\begin{array}{c}\text { ALB } \\
\left(\mathrm{mg} \mathrm{dl}^{-1}\right)\end{array}$ & $\begin{array}{c}\text { ACR } \\
\left(\mathrm{mg} \mathrm{g}^{-1}\right)^{\mathrm{b}}\end{array}$ & ALB & ACR \\
\hline Synthetic urine 1 & 30 & 300 & $32.36 \pm 0.12$ & $354.81 \pm 0.01$ & 92.13 & 81.73 \\
\hline Synthetic urine 2 & 50 & 500 & $54.95 \pm 0.56$ & $616.60 \pm 0.01$ & 90.10 & 76.68 \\
\hline Synthetic urine 3 & 100 & 1,000 & $102.33 \pm 0.09$ & $1,122.02 \pm 0.01$ & 97.67 & 87.80 \\
\hline Synthetic urine 4 & 400 & 4,000 & $407.38 \pm 0.44$ & $4,265.80 \pm 0.01$ & 98.16 & 93.38 \\
\hline
\end{tabular}

${ }^{a}$ Composed of ALB and CRE in artificial urine solution as shown in Table 1.

b-cratio ACR: mg ALB per gram CRE.

less than 5\%. These results suggested that the proposed method has relatively good accuracy and excellent precision, which is possible to be applied for practical applications in a clinical field.

\section{CONCLUSION}

Inexpensive, simple, fast, disposable, and affordable PADs were developed as an analytical tool used to determine the ACR in synthetic urine samples. The developed PADs can simultaneously measure ALB and CRE levels using a single microfluidic paper-based analytical device. In addition, this method allows a simple way to directly calculate the ACR, which can be possibly applied for early screening of nephropathy. In the future, this method should be examined for real urine samples since it can potentially be used as a medical diagnosis of a random urine sample without having to collect urine for 24 hours.

\section{ACKNOWLEDGMENTS}

A. Sabarudin would like to thank the Ministry of Finance of the Republic of Indonesia for financially supporting this work through RISPRO LPDP 2021 (PRJ-033/LPDP/2021).

\section{AUTHORS' CONTRIBUTIONS}

All authors made substantial contributions to the conception and design, acquisition of data, or analysis and interpretation of data; they took part in drafting the article or revising it critically for important intellectual content; agreed to submit to the current journal; gave final approval of the version to be published; and agreed to be accountable for all aspects of the work.

\section{CONFLICT OF INTERESTS}

The authors declare that no financial or other conflicts of interest.

\section{FUNDING}

This work was funded by the Ministry of Finance of the Republic of Indonesia through RISPRO LPDP 2021 (PRJ-033/ LPDP/2021).

\section{ETHICAL APPROVAL}

This study does not involve experiments on animals or human subjects.

\section{PUBLISHER'S NOTE}

This journal remains neutral with regard to jurisdictional claims in published institutional affiliation.

\section{REFERENCES}

Alila S, Boufi S, Belgacem MN, Beneventi D. Adsorption of a cationic surfactant onto cellulosic fibers I. surface change effects. Langmuir, 2005; 21(18):8106-13.

Brooks T, Keevil CW. A simple artificial urine for the growth of urinary pathogens. Lett Appl Microbiol, 1997; 24 (3):203-6.

Chagas CLS, Souza FR, Cardoso TMG, Moreira RC, Silva JAF, Jesus DP, Coltro WKT. A fully disposable paper-based electrophoresis microchip with integrated pencil-drawn electrodes for contactless conductivity detection. Anal Methods, 2016; 8:6682-6.

Chaiyo S, Kalcher K, Apilux A, Chailapakul O, Siangproh W. A novel paper-based colorimetry device for the determination of the albumin to creatinine ratio. Analyst, 2018; 143:5453-60.

Chapman DP, Gooding KM, McDonald TK, Shore AC. Stability of urinary albumin and creatinine after 12 months storage at $-20^{\circ} \mathrm{C}$ and $-80^{\circ} \mathrm{C}$. Pract Lab Med, 2019; 22(15):e00120.

Cook JGH. Factors influencing the assay of creatinine: prepared for the association of clinical biochemists' scientific and technical committee. Ann Clin Biochem, 1975; 12(36):219-32.

Corder CJ, Leslie SW. 24-hour urine collection. In: StatPearls, StatPearls Publishing, Treasure Island, FL, pp 242-5, vol 17(3), 2019 Available from: https://www.ncbi.nlm.nih.gov/books/NBK482482/

Delanghe JR, Speeckaert MM. Creatinine determination according to Jaffe--what does it stand for? Clin Kidney J, 2011; 4(2):83-6.

Fernandes A, de Souza PS, de Oliveira AE, Chaves AR. A new method for the determination of creatinine in urine samples based on disposable pipette extraction. J Braz Chem Soc, 2017; 29(4):695-700.

Han JS, Lee MJ, Park KS, Han SH, Yoo TH, Oh KH, Park SK, Lee J, Hyun YY, Chung W, Kim YH, Ahn C, Choi KH. Albuminuria as a risk factor for anemia in chronic kidney disease: result from the KoreaN cohort study for outcomes in patients with chronic kidney disease (KNOWCKD). PLoS One, 2015; 10(10):e0139747.

Heist CA, Bandara GC, Bemis DJ, Pommerenck JC, Remcho VT. New paper-based microfluidic tools for the analysis of blood serum protein and creatinine built via aerosolized deposition of polycaprolactone. Anal Methods, 2018; 10:2994-3000.

Hong DSC, Oh, IH, Park JS, Lee, CH, Kang CM, Kim GH Evaluation of urinary indices for albuminuria and proteinuria in patients with chronic kidney disease. Kidney Blood Press Res, 2016; 41(3):258-66.

Jalal UM, Jin GJ, Shim JS. Paper-plastic hybrid microfluidic device for smartphone-based colorimetric analysis of urine. Anal Chem, 2017; 89(24):13160-6.

Krishnegowda A, Padmarajaiah N, Anantharaman S, Honnur K. Spectrophotometric assay of creatinine in human serum sample. Arab J Chem, 2017; 10:S2018-24.

Küme T, Sağlam B, Ergon C, Sisman AR. Evaluation and comparison of Abbott Jaffe and enzymatic creatinine methods: could the old method meet the new requirements? J Clin Lab Anal, 2017; 32(1):e22168.

Martinez AW, Phillips ST, Carrilho E, Thomas III SW, Sindi H, Whitesides GM. Simple telemedicine for developing regions: camera 
phones and paper based microfluidic devices for real-time, off-site diagnosis. Anal Chem, 2018; 80(10):3699-707.

Martinez AW, Phillips ST, Butte MJ, Whitesides GM. Patterned paper as a platform for inexpensive, low-volume, portable bioassays. Angew Chem Int Ed, 2007; 46(8):1318-20.

Methven S, MacGregor MS, Traynor JP, O’Reilly DS, Deighan CJ. Assessing proteinuria in chronic kidney disease: protein-creatinine ratio versus albumin-creatinine ratio. Nephrol Dial Transplant, 2010; 25(9):2991-6.

Mohart V. Hypertension and chronic kidney diseases. Cor Vasa, 2013; 55(4):e397-402.

Neuen BL, Chadban SJ, Demaio AR, Johnson DW, Perkovic V. Chronic kidney disease and the global NCDs agenda. BMJ Glob Health, 2017; 2(2):E000380.

Ramanavicius A. Amperometric biosensor for the determination of creatine. Anal Bioanal Chem, 2007; 387(5):1899-906.

Sabarudin A. Sequential injection at valve mixing (SI-VM) for determination of albumin-creatinine ratio in urine. Orient J Chem, 2018; 34(2):730-4.

Schosinsky KH, Vargas M, Esquivel AL, Chavarria MA. Simple spectrophotometric determination of urinary albumin by dye-binding with use of bromphenol blue. Clin Chem, 1987; 33(1):223-6.

Shaw KM, Cummings MH. Diabetes: chronic complications. 3rd edition, Wiley-Blackwell, Hoboken, NJ, pp 34-66, 2012.

Shiba N, Shimokawa H. Chronic kidney disease and heart failure - bidirectional close link and common therapeutic goal. J Cardiol, 2011; 57(1):8-17.

Siangproh W, Teshima N, Sakai T, Katoh S, Chailapakul O. Alternative method for measurement of albumin/creatinine ratio using spectrophotometric sequential injection analysis. Talanta, 2009; 79(4):1111-7.

Simerville JA, Maxted WC, Pahira JJ. Urinalysis: a comprehensive review. Am Fam Physician, 2005; 71(6):1153-62.

Van Buren PN, Toto R. Hypertension in diabetic nephropathy: epidemiology, mechanisms, and management. Adv Chronic Kidney Dis, 2011; 18(1):28-41.

Yamada K, Henares TG, Suzuki K, Citterio D. Paper-based inkjet printed mikrofluidic analytical devices. Angew Chem, 2015; 54:2-19.

Yuen PST, Dunn SR, Miyaji T, Yasuda H, Sharma K, Star RA. Simplified method for HPLC determination of creatinine in mouse serum. Am J Physiol Renal Physiol, 2004; 286(6):F1116-9.

Suzuki Y. Reaction between $\mathrm{pH}$ indicators with a large $\mathrm{pKa}$ value and human serum albumin and its application to the determination of the serum albumin concentration. Bunseki Kagaku, 2002; 52(10):939-44.

\section{How to cite this article:}

Nurrahmah N, Amalia KT, Sulistyarti H, Sabarudin A. Fast colorimetric detection of albumin-to-creatinine ratio using paper-based analytical devices with alkaline picrate and Bromothymol Blue reagents. J Appl Pharm Sci, 2022; 12(01):140-148. 